\title{
Individual cognitive and depressive traits associated with maternal versus paternal family history of Late-onset Alzheimer's disease: Proactive semantic interference versus standard neuropsychological assessments
}

\author{
Kathleen E. Wilson ${ }^{\mathrm{a}}$, Carolina Abulafiaa, ${ }^{\mathrm{a}, \mathrm{b}}$, David A. Loewenstein ${ }^{\mathrm{d}}$, Daniel E. Vigo ${ }^{\mathrm{b}, \mathrm{c}}$, \\ Gustavo Sevlever $^{\mathrm{e}}$, Charles B. Nemeroff ${ }^{\mathrm{d}}$, Mirta F. Villarreal ${ }^{\mathrm{a}, \mathrm{c}, \mathrm{f}}$, Salvador M. Guinjoan ${ }^{\mathrm{a}, \mathrm{c}, \mathrm{g}, \mathrm{h}, \mathrm{i}, *}$ \\ ${ }^{a}$ Grupo de Investigación en Neurociencias Aplicadas a las Alteraciones de la Conducta, Fundación FLENI, Buenos Aires, Argentina \\ b Chronophysiology Laboratory, Institute for Biomedical Research (UCA - CONICET), Buenos Aires, Argentina \\ ${ }^{\mathrm{c}}$ Consejo Nacional de Investigaciones Científicas y Técnicas, Buenos Aires, Argentina \\ ${ }^{\mathrm{d}}$ Department of Psychiatry and Behavioral Sciences and Center on Aging, Miller School of Medicine, University of Miami, United States of America \\ e Departamento de Neuropatología y Biología Molecular, Fundación FLENI, Buenos Aires, Argentina \\ ${ }^{\mathrm{f}}$ Department of Physics (FCEyN), University of Buenos Aires, Argentina \\ ${ }^{8}$ FLENI Teaching Unit, Department of Psychiatry and Mental Health, University of Buenos Aires School of Medicine, Buenos Aires, Argentina

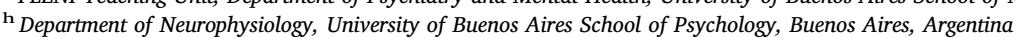 \\ ${ }^{\text {i }}$ Servicio de Psiquiatría, Fundación FLENI, Buenos Aires, Argentina
}

\section{A R T I C L E I N F O}

\section{Keywords:}

Early diagnosis

Late-onset Alzheimer's disease

Loewenstein-Acevedo Scale for Semantic

Interference and Learning (LASSI-L)

Rey Auditory Visual Learning Test (RAVLT)

\begin{abstract}
A B S T R A C T
The main objective of this study was to assess cognitive and depressive manifestations associated with Alzheimer's disease in middle-aged asymptomatic individuals with maternal versus paternal family history of the disease using standardly used neuropsychological measures and a novel cognitive stress test, the LoewensteinAcevedo Scale for Semantic Interference and Learning (LASSI-L). We evaluated cognitive abilities in offspring of late-onset Alzheimer's disease patients and a control group with no family history of dementia. Results showed lower cognitive performance in verbal episodic memory and semantic memory in participants with an Alzheimer's parent, especially in individuals with a maternal family history of the disease. While the standardly used neuropsychological evaluations were sensitive to differences in cognition between those with a maternal history of the disease and the control group, the LASSI-L was sensitive to proactive semantic interference impairments in both groups with a paternal and maternal family history of the disease. Depressive symptoms may affect cognitive performance differently in individuals with a family history of Alzheimer's disease compared to a control group, especially in individuals with a maternal history of the disease. This study highlights the value of semantic interference paradigms in early detection of Alzheimer's and emphasizes the importance of studying maternal versus paternal transmission of the disease.
\end{abstract}

\section{Introduction}

Alzheimer's disease is the most common form of dementia, accounting for $60-80 \%$ of dementia cases worldwide [1]. Late-onset Alzheimer's disease (LOAD) develops in patients aged over 65 years, comprises at least $95 \%$ of all Alzheimer's cases and is associated with confusion, memory problems, and behavioral changes [2,3]. Alzheimer's disease is characterized by two neuropathological hallmarks: extracellular plaques of amyloid beta (A $\beta$ ), which block cell-to-cell signaling, and intracellular Tau tangles, which impair the intracellular transport system, both of which cause cell death and lead to atrophy of several brain regions, including the cerebral cortex and the hippocampus [4].

Previous studies have evaluated cognitive phenotypes associated with late-onset Alzheimer's disease in asymptomatic individuals who are at-risk for developing the disease and identified specific patterns of cognitive decline beginning years before diagnosis of the disease, including impairments in episodic and semantic memory, executive functioning, and verbal intelligence [5-7]. A review of a longitudinal cohort and neuroimaging studies to determine correlations between

\footnotetext{
* Corresponding author at: Servicio de Psiquiatría, Fundación FLENI, Montañeses 2325 5th floor C1428AQK, Buenos Aires, Argentina. Tel.: + 541157773200 $\mathrm{x} 2514 / 2531$.

E-mail address: sguinjoan@fleni.org.ar (S.M. Guinjoan).
} 
brain biomarkers and specific cognitive functions in preclinical Alzheimer's disease showed that episodic memory was the most salient cognitive deficit, correlating with hypoconnectivity across large-scale brain networks and high levels of amyloid deposition in individuals with preclinical Alzheimer's disease [8].

After age, a family history of Alzheimer's disease is the second greatest risk factor for developing the disease [9,10]. Recent studies have suggested that the sex of the parent with Alzheimer's disease may also impact the development of Alzheimer's disease in their offspring, with some evidence in epidemiological studies indicating that maternal transmission may be more prevalent compared to paternal transmission of the disease [11]. Other studies have also shown that children of mothers with Alzheimer's disease have 3-9 times higher risk of developing Alzheimer's than those of fathers with the disease and that approximately $20 \%$ of all LOAD cases are maternally inherited - one study reporting a prevalence of $71.86 \%$ of maternal transmission compared to $28.14 \%$ of paternal transmission in a sample of subjects with family history of AD $[12,13]$. Some authors argue that maternally/inherited $\mathrm{AD}$ represents a different phenotype since these subjects present an earlier onset, lower scores on cognitive test and more pronounced ADrelated brain abnormalities such as reduced brain metabolism, higher $\mathrm{A} \beta$ burden and decreased gray matter volume). Some of the suggested genetic mechanisms for maternal transmission are chromosome $\mathrm{X}$ mediated transmission, genomic imprinting and through mitochondrial DNA transmission [14].

Depression may also play an important role in Alzheimeŕs disease because previous studies have shown that depressive symptoms are increased in preclinical Alzheimeŕs [15], depressed mood moderately increases the risk of developing Alzheimer's disease and other dementias [16], and the risk of dementia increases as the number of episodes of depression and bipolar affective disorder increases [17]. There is also evidence of a correlation between the Apolipoprotein e3/ e4 genotype, a risk factor for Alzheimer's disease, and late-onset major depression [18]. Additionally, the prevalence of lifetime major depression is twice as high in women compared to men, making it a particularly important variable to study in relation to maternal versus paternal transmission of Alzheimeŕs disease [19]. Elevated anxiety symptoms in individuals with preclinical Alzheimeŕs disease have also been shown to play a role in the relationship between amyloid deposition and cognitive decline [20].

The objective of the present investigation was to determine if there are differences in cognition and depression in midldle-aged, asymptomatic individuals with a maternal history or paternal history of LOAD compared to a group of middle-aged individuals with no family history of dementia. In addition to a standardly used neuropsychological assessment battery, this study evaluated participants with a novel semantic interference paradigm using the Loewenstein-Acevedo Scale for Semantic Interference and Learning (LASSI-L), which has shown higher levels of sensitivity and specificity in discriminating between individuals with amnestic mild cognitive impairment (MCI) and healthy elderly individuals compared to standardly used neuropsychological measures of memory function [21]. Given the elevated sensitivity of this semantic interference test, we predicted that the LASSI-L would discriminate between individuals with no family history of dementia and individuals at-risk for Alzheimer's disease in both the maternal and paternal history groups, whereas standardly used neuropsychological measures would only be sensitive to discriminate cognitive deficits between at-risk individuals of Alzheimer's disease compared to the control group. In addition, we predicted that depressive symptoms would correlate with cognitive function differently in individuals with a family history of the disease compared to controls without a family history of dementia.

\section{Methods}

\subsection{Design and sample}

This was a cross-sectional study, where cognitive measures were compared between a sample of participants with a maternal family of Alzheimer's disease (FHm), a paternal family of Alzheimer's disease (FHp), and control subjects with no family history of dementia (CS). The study protocol was performed in accordance with the Declaration of Helsinki and approved by the Bioethics Committee of FLENI Foundation, Argentina. All participants provided their written informed consent for the study. The sample size consisted of 22 individuals with a maternal history of Alzheimer's disease, 13 individuals with a paternal history of Alzheimer's disease, and 25 control subjects with no family history of dementia. The three groups were comparable in gender, age, and education level. The inclusion criteria for the offspring of participants with Alzheimer's disease were as follows: (1) one parent diagnosed with probable LOAD according to DSM-5, (2) 40-65 years of age at the time of recruitment, and (3) seven or more years of formal education. The control group had the same inclusion criteria except for a family history of AD. The exclusion criteria for all groups were as follows: (1) Mini Mental State Examination (MMSE) score < 25, (2) compromised intellectual level based on education and employment history, (3) evidence of current progressive neurological disease or likely to impair cognitive performance, (4) current and prior history of substance abuse (alcohol, marijuana, stimulants, benzodiazepines, or other drugs), and (5) Hachinski score $>7$ (to exclude individuals with vascular-derived cognitive impairment).

\subsection{Cognitive assessment}

The methods describing the standardly used cognitive assessments in this study are described elsewhere [22]. The standardly used neuropsychological tests selected for this study have been widely validated and are frequently used in clinical practice. They consist of a concise battery aimed at assessing the most salient cognitive domains impaired in Alzheimer's disease: episodic memory and language. The neuropsychological evaluation was performed in a single session of approximately $90 \mathrm{~min}$ by an experienced neuropsychologist. The MMSE [23] and the Clock Drawing Test subtest from the 7 Minute Screen test [24] were included as screening measurements. Although the MMSE has been shown to be insensitive to prodromal Alzheimer's disease, we incorporated it as a widely used screening instrument [25] to be complemented with more sensitive tests. Verbal episodic memory was assessed by the Rey Auditory Verbal Learning Test [26,27]. Semantic memory was assessed by the semantic fluency task ("animals" category) [28], the Vocabulary subtest of the intelligence battery WAIS-III [29] and the Boston naming test [30]. Other verbal intelligence evaluations included an Argentine accentuation reading test of words (TAP-BA) to assess premorbid intelligence [31] and a phonologic fluency evaluation (letter "p") [32].

The Loewenstein-Acevedo Scale for Semantic Interference and Learning (LASSI-L) was designed to target the vulnerability to semantic interference in individuals with mild cognitive impairment at risk for developing Alzheimer's disease and is described in detail elsewhere [33]. The LASSI-L consists of two word lists (list A and list B), each with 15 words and 3 semantic categories: fruits, musical instruments, and articles of clothing. The investigator instructs the participant to read each word aloud as each word is presented individually. After reading all 15 words, the investigator then asks for the participant to recall as many of the words from the list as possible (free recall). The investigator then gives semantic cues for each category and asks for the 
participant to recall as many words as possible in each semantic category (cued recall). The investigator then instructs the participant to repeat the exercise using the same list of words for a second learning trial. Participants are then presented again with semantic category cues and asked to recall words by category. The next part of the evaluation consists of the same tasks as the first part of the evaluation and uses the same 3 semantic categories, but with a different list of 15 words, eliciting proactive interference. An additional trial of List B assesses failure to recover from proactive interference (frPSI). Finally, without presenting the first list of words again, the investigator asks for a free recall followed by a cued recall of list A, evaluating retroactive interference. In the delayed recall section, participants are asked to recall words from both lists combined.

Some of the key features of the LASSI-L that differentiate it from standardly used neuropsychological evaluations include (1) explicit identification of the semantic categories used, (2) the use of a second list of semantically related target words, (3) using the same category cues for semantic interference and for the elicitation of semantic errors, and (4) evaluation of the failure to recover from proactive semantic interference (frPSI). For this investigation we focused on cued recall A2 (maximum storage and retrieval), B1 cued recall (susceptible to PSI), B2 cued recall (susceptible to frPSI) and short-delayed cued recall (subject to retroactive semantic interference (rSI). The LASSI-L cued learning score was calculated by averaging the scores of each semantic category on the cued recall portions of List A.

Because of the exploratory nature of the present study and one of its aims, which is the detection of subtle cognitive changes in comparable groups (age, sex and education level) of healthy middle-aged asymptomatic subjects, all cognitive tests measures were reported and analyzed as raw scores. Standardized scores in this case would obscure the detection of such small changes, which do not even constitute clinical deficits in performance given our sample's characteristics.

The Cognitive Reserve Questionnaire (CRQ) was administered to all participants to assess the most relevant elements associated to cognitive reserve (education level, parent's education level, additional academic courses completed, professional activity, musical education, fluent languages, reading activity, and ingenuity games) [34]. The concept of cognitive reserve refers to how individuals who engage in enriching cognitive activities may have a greater resistance to cognitive aging and dementia compared to those who do not have this enrichment [35]. The presence and severity of depressive symptoms was measured by the Beck Depression Inventory-II (self-report) and by the Hamilton Depression Rating Scale (HDRS), completed by a clinician. All participants were cognitively asymptomatic and their neuropsychological testing yielded normal values; none of the individuals met criteria for mild cognitive impairment or dementia.

\subsection{Statistical analysis}

Continuous variables were summarized by means and standard deviation. Categorical variables were summarized as frequencies and percentages. Differences in demographic and clinical data, standardly used cognitive assessments, and LASSI-L scores between groups were calculated by using Analysis of Variance (ANOVA). If significant main effects were found, pairwise difference was calculated using post hoc analysis by Tukey HSD tests. LASSI-L intrusion scores ( $>1$ intrusion, i.e. the proportion of participants with more than one intrusion) were calculated by means of the chi-square procedure. Correlations between clinical data and cognitive variables were evaluated using Pearson's correlation coefficients. We report two-tailed significance at $p<.05$. A false discovery rate was applied using the online SDM project false discovery rate (FDR) calculator for $p$ values on Tables 1-3 [36]. Bolded $p$ values indicate survival of the false discovery rate calculation. Kolmogorov-Smirnov test was performed to confirm normal distribution in all three sample groups. All statistical analysis was performed using SPSS version 22.0 software (SPSS Inc.).

\section{Results}

\subsection{Demographic and clinical data}

Table 1 shows the demographic and clinical characteristics of the subjects with maternal family history of Alzheimer's disease (FHm), paternal family history of Alzheimer's disease (FHp), and a control group with no family history of dementia (CS). The three groups were comparable in age and sex, as well as in education, chronotype, depression (as measured by the Hamilton Depression Rating Scale and Beck Depression Inventory-II), and Hachinski score (Table 1). Participants with a family history of Alzheimer's disease had lower cognitive reserve scores as compared to those without a family history of dementia, but this difference did not reach significance after adjustment of $p$ values for the false discovery rate [FDR] (Table 1). The level of education of the parents of participants was not significantly different among the three groups (Table 1).

\subsection{Standardly used neuropsychological evaluations}

Table 2 shows significant differences between groups on standardly used cognitive assessments. Participants with a family history of Alzheimer's disease scored lower on several standardly used neuropsychological tests compared to the control group, including the Mini Mental State Exam (MMSE), several sections of the Rey Auditory Verbal Learning Test (RAVLT), and the vocabulary section of the Wechsler Intelligence Scale- III (WAIS-III) (Table 2).

Post hoc analysis showed participants with a maternal history of Alzheimer's disease scored significantly worse on several sections of the Rey Auditory Verbal Learning Test (RAVLT), including the delayed memory $(p=.003)$, recognition $(p=.023)$, and $\%$ retention $(p=.007)$ scores, as well as the WAIS-III vocabulary test $(p=.003)$, compared to the control group (Table 2). These differences on the RAVLT and the WAIS-III Vocabulary test were not observed among participants with a paternal history of the disease and the control group, or between participants with a maternal versus paternal history of Alzheimer's disease. Post hoc analysis also showed participants with a paternal history of Alzheimer's disease scored significantly lower than the control group on the Mini Mental State Exam $(p=.045)$, though this difference was not observed between the control group and those with a maternal history of the disease. There were no significant differences in cognition between participants with a maternal versus paternal history of Alzheimer's disease (Table 2).

\subsection{LASSI-L}

Participants with a family history of Alzheimer's disease performed worse than controls on the LASSI-L (Table 3). Both groups with a family history of Alzheimer's had significantly more B2 cued intrusions than controls $\left(x^{2}=11.84, p=.003\right)$. Individuals with a maternal history of the disease exhibited more intrusion errors on several sections of the LASSI-L compared to individuals with a paternal history of Alzheimer's disease and the control group, though these differences did not survive correction for multiple comparisons (Table 3).

\subsection{Correlations between depressive symptoms and cognition}

We sought to determine if there were correlations between the Hamilton Depression Rating Scale (HDRS) and performance on several cognitive evaluations. There was a significant negative relationship between the Hamilton Depression Rating Scale and the Mini Mental State Exam (MMSE) in the control group $(r=-0.493, p=0.020)$. In individuals with a maternal history of Alzheimer's disease, there was a negative relationship between the Hamilton Depression Rating Scale and the LASSI-L cued learning measure $(\mathrm{r}=-0.685, p=0.014)$. There were no significant correlations between the Hamilton Depression 
Table 1

Demographic and clinical data.

\begin{tabular}{|c|c|c|c|c|c|c|c|c|c|c|c|}
\hline & \multicolumn{3}{|c|}{ Controls } & \multicolumn{3}{|c|}{ Maternal History of $\mathrm{AD}$} & \multicolumn{3}{|c|}{ Paternal History of $\mathrm{AD}$} & \multirow[t]{2}{*}{ f } & \multirow[t]{2}{*}{$\mathrm{p}$} \\
\hline & $\mathrm{N}$ & Mean or Frequency & SD or $\%$ & $\mathrm{~N}$ & Mean or Frequency & SD or $\%$ & $\mathrm{~N}$ & Mean or $\%$ & SD & & \\
\hline Age & 25 & 51.17 & 8.223 & 22 & 54.45 & 9.354 & 13 & 52.69 & 6.473 & 0.892 & 0.416 \\
\hline Female & 25 & 21 & $76 \%$ & 22 & 18 & $72.7 \%$ & 13 & $53.8 \%$ & 6 & 2.608 & 0.082 \\
\hline Education (years) & 25 & 18.17 & 3.200 & 22 & 16.76 & 2.705 & 13 & 18.00 & 3.536 & 1.076 & 0.349 \\
\hline Cognitive Reserve & 24 & 17.88 & 2.787 & 22 & $15.18^{*}$ & 3.390 & 11 & 15.91 & 3.081 & 4.58 & 0.015 \\
\hline Cognitive Reserve- Parent Education & 24 & 1.63 & 0.495 & 22 & 1.64 & 0.492 & 11 & 1.82 & 0.405 & 0.687 & 0.507 \\
\hline Chronotype (ICSP) & 17 & 37.82 & 10.870 & 20 & 36.75 & 7.489 & 9 & 34.11 & 10.105 & 0.465 & 0.631 \\
\hline Hachinski Ischemic Score & 20 & 0.85 & 1.040 & 21 & 1.24 & 1.221 & 11 & 5.27 & 14.227 & 1.846 & 0.169 \\
\hline Beck Depression Inventory-II & 20 & 7.55 & 7.598 & 20 & 9.45 & 6.970 & 11 & 7.09 & 5.262 & 0.556 & 0.577 \\
\hline Hamilton Depression & 22 & 7.18 & 5.439 & 22 & 9.09 & 7.584 & 11 & 7.09 & 5.873 & 0.594 & 0.556 \\
\hline
\end{tabular}

(") Indicates a significant difference of $\mathrm{p}<.05$ between controls and one group of participants with a family history of Alzheimer's disease.

Rating Scale and LASSI-L scores in individuals with a paternal history of Alzheimer's disease.

\section{Discussion}

The purpose of this study was to evaluate an Alzheimer-associated cognitive phenotype in asymptomatic, middle-aged offspring of Alzheimer's patients and to investigate the development and progression of cognitive impairment in at-risk individuals based on the sex of the parent with the disease. Furthermore, we wanted to investigate if certain cognitive measures were more sensitive in differentiating between subtle cognitive deficits in asymptomatic, at-risk individuals compared to a control group with no family history of dementia and moreover if depressive symptom severity had a negative effect on cognitive performance in preclinical Alzheimer's disease.

The results support our hypothesis that that the sex of the parent with Alzheimer's disease may modify the cognitive abilities of asymptomatic, middle-aged offspring when compared to a control group without family history of the disease. These results highlight the deficits in verbal episodic and semantic memory in individuals with a maternal history of Alzheimer's disease and suggest that a standardly used battery of neuropsychological assessment is sufficient in detecting these subtle cognitive differences between offspring of mothers with Alzheimer's disease and a control group, but not between those with a paternal history of the disease and a control group with no family history of dementia.

Neuroimaging studies indicate that the progression of Alzheimer's may be different in individuals with a maternal family history of the disease, including hypometabolism of regions typically affected by Alzheimer's in FDG-PET scans of offspring of mothers with $\mathrm{AD}$ compared to offspring of fathers with Alzheimer's disease and a control group with no family history of the disease $[37,38]$. Additionally, individuals with a maternal history of Alzheimer's disease have greater atrophy in brain regions susceptible to $\mathrm{AD}$ compared to those with a paternal history of the disease and a control group [39]. One interpretation of these studies is that a maternal history of AD may lower the age of disease onset in the offspring [40]. Many of the traditional neuropsychological assessments were originally developed to evaluate dementia but are not necessarily optimal in evaluating the earliest stages of cognitive impairment in preclinical Alzheimer's disease [21]. However, a lower age of AD onset in individuals with a maternal history of Alzheimer's may explain why some of the traditional neuropsychological assessments detected subtle but significant cognitive differences between individuals with a maternal history of $\mathrm{AD}$ and controls but not between those with a paternal history of $\mathrm{AD}$ and the control group in the present study.

The obtained results support our hypothesis that the LASSI-L is more sensitive to subtle cognitive impairments in asymptomatic, at-risk individuals with a family history of Alzheimer's disease, regardless of maternal or paternal family history of the disease. As discussed elsewhere [21], the LASSI-L provides unique advantages for detecting cognitive deficits in semantic interference, which may reflect initial manifestation of the disease in at-risk individuals. The significant difference in cued intrusions for List B between both groups with a family history of Alzheimer's disease and a control group suggests that the LASSI-L is particularly sensitive to the subtle deficits in tasks involving proactive semantic interference, which may indicate the earliest manifestations of the disease.

A previous study evaluating the utility of the LASSI-L in early detection for Alzheimer's disease found that individuals with amnestic mild cognitive impairment (aMCI) had greater proactive and retroactive interference compared to normal elderly subjects [21]. Specifically, the second cued recall for both List A and List B were the strongest predictors of aMCI in logistic regression models and had a greater discriminatory power relative to delayed memory for passages, suggesting that evaluating semantic interference may be more powerful in detecting early features of Alzheimer's disease compared to other evaluations [21]. Other studies have found equivalent yet subtler semantic interference difficulties in asymptomatic middle-aged offspring of patients with Alzheimer's disease and such reduced performance was associated to structural changes in AD-relevant regions, increased amyloid load in the temporal lobe [41] and also exhibited inverse correlations with functional connectivity in limbic regions [42], providing evidence that deficits in semantic interference may represent

Table 2

Standardly used neuropsychological tests.

\begin{tabular}{|c|c|c|c|c|c|c|c|c|c|c|c|}
\hline & \multicolumn{3}{|c|}{ Controls } & \multicolumn{3}{|c|}{ Maternal History of $\mathrm{AD}$} & \multicolumn{3}{|c|}{ Paternal History of $\mathrm{AD}$} & \multirow[t]{2}{*}{$\mathrm{f}$} & \multirow[t]{2}{*}{$\mathrm{p}$} \\
\hline & $\mathrm{N}$ & Mean & SD & $\mathrm{N}$ & Mean & SD & $\mathrm{N}$ & Mean & SD & & \\
\hline Mini Mental State Exam & 24 & 29.63 & 0.711 & 22 & 29.00 & 0.976 & 12 & $28.75^{*}$ & 1.485 & 3.761 & 0.029 \\
\hline RAVLT Delayed Memory & 23 & 11.00 & 2.111 & 21 & $8.19^{*}$ & 2.943 & 12 & 9.67 & 3.143 & 6.044 & 0.004 \\
\hline RAVLT Recognition & 23 & 14.17 & 1.193 & 21 & $12.81^{*}$ & 2.182 & 12 & 13.33 & 1.303 & 3.791 & 0.029 \\
\hline RAVLT Retention & 23 & 93.71 & 16.899 & 21 & $75.13^{*}$ & 22.068 & 12 & 83.563 & 18.288 & 4.981 & 0.010 \\
\hline WAIS-III Vocabulary & 20 & 52.30 & 5.253 & 22 & $44.18^{*}$ & 8.198 & 12 & 46.67 & 9.509 & 6.163 & 0.004 \\
\hline
\end{tabular}

( $)$ Indicates a significant difference of $\mathrm{p}<.05$ between controls and one group of participants with a family history of Alzheimer's disease. 
Table 3

LASSI-L measures.

\begin{tabular}{|c|c|c|c|c|c|c|c|c|c|c|c|}
\hline & \multicolumn{3}{|c|}{ Controls } & \multicolumn{3}{|c|}{ Maternal History of $\mathrm{AD}$} & \multicolumn{3}{|c|}{ Paternal History of $\mathrm{AD}$} & \multirow[t]{2}{*}{ Statistic } & \multirow[t]{2}{*}{$\mathrm{P}$} \\
\hline & $\mathrm{N}$ & Mean or Frequency & SD or $\%$ & $\mathrm{~N}$ & Mean or Frequency & SD or $\%$ & $\mathrm{~N}$ & Mean or Frequency & $\mathrm{SD}$ or $\%$ & & \\
\hline A2 Cued Recall & 21 & 14.19 & 1.030 & 12 & 13.83 & 1.030 & 10 & 13.60 & 1.265 & $\mathrm{f}=1.100$ & 0.343 \\
\hline A2 Cued Intrusions & 21 & 0.24 & 0.539 & 12 & 0.42 & 0.900 & 10 & 0.20 & 0.422 & $f=0.398$ & 0.674 \\
\hline$>1$ A2 Cued Intrusions & 21 & 1 & 0.0476 & 12 & 0.05 & 0.218 & 10 & 0 & 0 & $\mathrm{X}^{2}=0.855$ & 0.652 \\
\hline B1 Cued Recall & 21 & 7.90 & 2.982 & 12 & 7.08 & 2.353 & 10 & 8.00 & 2.494 & $f=0.653$ & 0.430 \\
\hline B1 Cued Intrusions & 21 & 0.95 & 1.322 & 12 & 2.50 & 3.030 & 10 & 1.90 & 1.853 & $f=2.329$ & 0.111 \\
\hline$>1 \mathrm{~B} 1$ Cued Intrusions & 21 & 6 & 0.2857 & 12 & 6 & 0.5 & 10 & 4 & 0.4 & $\mathrm{X}^{2}=1.544$ & 0.462 \\
\hline B2 Cued Recall & 21 & 12.24 & 1.700 & 12 & 11.42 & 1.505 & 10 & 11.30 & 1.494 & $f=1.612$ & 0.212 \\
\hline B2 Cued Intrusions & 21 & 0.62 & 0.590 & 12 & 1.67 & 1.969 & 10 & 1.60 & 1.075 & $f=3.713$ & 0.033 \\
\hline$>1 \mathrm{~B} 2$ Cued Intrusions & 21 & 1 & 0.0476 & 12 & $5^{*}$ & 0.4167 & 10 & $6^{*}$ & 0.6 & $X^{2}=11.84$ & 0.003 \\
\hline
\end{tabular}

$\left(^{*}\right)$ Indicates a significant difference of $\mathrm{p}<.05$ between controls and at least one group of participants with a family history of Alzheimer's disease.

structural and limbic circuit dysfunction in early pathophysiology of Alzheimer's disease and that the LASSI-L should therefore be especially sensitive to subtle cognitive impairments in individuals with a family history of the disease. These studies support our findings that the LASSI$\mathrm{L}$ can detect subtle cognitive differences between both maternal and paternal history of $\mathrm{AD}$ compared to a control group, as this tool was specifically designed to target the specific memory deficits in prodromal $\mathrm{AD}$.

The negative correlations between the Hamilton Depression Rating Scale scores and cognitive assessments supported our hypothesis that depressive symptoms would have a different impact on cognition in individuals with a family history of Alzheimeŕs disease compared to the control group. Depressive symptoms correlated inversely with the MMSE in the control group, but not in individuals with a family history of Alzheimer's disease. Depressive symptoms correlated inversely with LASSI-L cued learning in individuals with a maternal history of Alzheimer's disease, but not in those with a paternal history of the disease or the control group, suggesting that depression may affect cognitive performance differently depending on the family history and sex of the parent with Alzheimer's. Previous studies also found that depression may affect cognition in preclinical and clinical Alzheimer's disease, as individuals with Alzheimer's disease but without depression tended to perform better on cognitive assessments than those with Alzheimer's disease and depression [43].

\section{Conclusion}

The results of this study confirm and extend previous observations that asymptomatic, middle-aged offspring of patients with Alzheimer's disease have subtle cognitive impairments compared to control subjects without a family history of the disease, including deficits in verbal episodic memory and semantic memory. These findings also support our hypothesis that standardly used neuropsychological tests highlight differences in cognition between individuals with a maternal inheritance of Alzheimer's disease and those without a family history of dementia, while the LASSI-L evaluation is more sensitive to cognitive impairments in both groups with a family history of Alzheimer's disease. The apparent increased cognitive deficits in at-risk individuals with a maternal history of the disease adds to the growing literature that the effects of Alzheimer's disease may differ depending on whether the disease is maternally or paternally inherited. Additionally, the sensitivity of the LASSI-L to proactive semantic interference in both groups with a family history of this disease provides further evidence that the LASSI-L can be a useful tool in research on early Alzheimer's disease manifestations. The correlations between depressive symptoms and cognition also suggest that depression may have a role in cognitive ability in individuals at-risk for developing Alzheimer's disease and may reflect an early phenotype of the disease, especially among those with maternal inheritance. However our sample size does not permit a definitive response to this issue which is open to further investigation.
One limitation of this study is the small sample size, especially in the number of participants with paternal inheritance. Whereas maternal inheritance revealed to exert a greater impact on cognitive performance than offspring with paternal inheritance - which is in line with the literature - it should be noted that any lack of influence on the paternal side could simply reflect inadequate statistical power due to the small number of cases for this sample group $(n=13)$. This becomes an important limitation in our study, since it was probably not powered enough to answer this essential question. In this regard, whereas results of offspring with maternal inheritance were compared to those with paternal transmission, we need to be careful when extrapolating these conclusions to the LOAD population because the lack of statistical power of the low sample size of the second O-LOAD might be hindering the detection of clinically meaningful differences. Apart from the issue with the O-LOAD with paternal inheritance sample size, an overall larger sample size is important for replication of these findings. A greater amount of subjects would allow for the analysis of LOAD related phenotypes in asymptomatic, at-risk individuals by both the sex of the parent with Alzheimer's disease and the sex of the participant, which may help us identify further sex differences in the disease and its transmission.

Future work for this study includes combining the current results with structural and functioning brain imaging data to further define neurodevelopmental characteristics associated with the Alzheimer's disease phenotype in offspring of patients with the disease and to determine if these results vary by the sex of the parent with the disease.

\section{Financial disclosures}

Research/Grants:

National Institutes of Health (NIH), Stanley Medical Research Institute

Consulting (last three years):

Xhale, Takeda, Taisho Pharmaceutical Inc., Prismic Pharmaceuticals, Bracket (Clintara), Total Pain Solutions (TPS), Gerson Lehrman Group (GLG) Healthcare \& Biomedical Council, Fortress Biotech, Sunovion Pharmaceuticals Inc., Sumitomo Dainippon Pharma, Janssen Research \& Development LLC, Magstim, Inc., Navitor Pharmaceuticals, Inc.

Stockholder:

Xhale, Celgene, Seattle Genetics, Abbvie, OPKO Health, Inc., Bracket Intermediate Holding Corp., Network Life Sciences Inc., Antares

Scientific Advisory Boards:

American Foundation for Suicide Prevention (AFSP), Brain and Behavior Research Foundation (BBRF) (formerly named National Alliance for Research on Schizophrenia and Depression [NARSAD]), Xhale, Anxiety Disorders Association of America (ADAA), Skyland Trail, Bracket (Clintara), RiverMend Health LLC, Laureate Institute for Brain Research, Inc. 
Board of Directors:

AFSP, Gratitude America, ADAA

Income sources or equity of $\$ 10,000$ or more:

American Psychiatric Publishing, Xhale, Bracket (Clintara), CME

Outfitters, Takeda

Patents:

Method and devices for transdermal delivery of lithium (US 6,375,990B1)

Method of assessing antidepressant drug therapy via transport inhibition of monoamine neurotransmitters by ex vivo assay (US

7,148,027B2)

Speakers Bureau:

None.

\section{Appendix A. Supplementary data}

Supplementary data to this article can be found online at https:// doi.org/10.1016/j.pmip.2018.09.002.

\section{References}

[1] Alzheimer's Association. Alzheimer's association report: 2017 Alzheimer's disease facts and figures. Alzheimer's Dementia 2017;2017(13):325-73.

[2] Reitz C, Brayne C, Mayeux R. Epidemiology of Alzheimer disease. Nat Rev Neurol 2012;7(3):137-52.

[3] Isik AT. Late onset Alzheimer's disease in older people. Clin Interv Aging 2010;5:307-11.

[4] Serrano-Pozo A, Frosch MP, Masliah E, Hyman BT. Neuropathological Alterations in Alzheimer Disease. Cold Spring Harb Perspect Med 2011;1(1):a006189.

[5] Chen P, Ratcliff G, Belle SH, Cauley JA, DeKosky ST, Ganguli M. Patterns of cognitive decline in presymptomatic Alzheimer's disease: a prospective community study. Arch Gen Psychiatry 2001;58(9):853-8.

[6] Grober E, Hall CB, Lipton RB, Zonderman AB, Resnick SM, Kawas C. Memory impairment, executive dysfunction, and intellectual decline in preclinical Alzheimer's disease. J Int Neuropsychol Soc 2008;14(2):266-78.

[7] Salmon DP. Neuropsychological features of mild cognitive impairment and preclinical Alzheimer's disease. Curr Top Behav Neurosci 2012;10:187-212.

[8] Mortamais M, Ash JA, Harrison J, et al. Detecting cognitive changes in preclinical Alzheimer's disease: A review of its feasibility. Alzheimer's Dementia 2017;13(4):468-92.

[9] Farrer LA, Cupples LA, Haines JL, Hyman B, et al. Effects of age, sex, and ethnicity on the association between apolipoprotein E genotype and Alzheimer disease. JAMA 1997;278:1349-56.

[10] Silverman JM, Raiford K, Edland S, Fillenbaum G, et al. The consortium to establish a registry for Alzheimer's disease (CERAD). Part VI. Family history assessment: A multicenter study of first degree relatives of Alzheimer's disease probands and nondemented spouse controls. Neurol 1994;44:1253-9.

[11] Edland SD, Silverman JM, Peskind ER, Tsuang D, et al. Increased risk of dementia in mothers of Alzheimer's disease cases: Evidence for maternal inheritance. Neurol 1996; 47:254-6.

[12] Gomez-Tortosa E, Barquero MS, Baron M, Saint HJ, et al. Variability of age at onset in siblings with familial Alzheimer disease. Arch Neurol 2007;64:1743-8.

[13] Mosconi L, et al. Maternal transmission of Alzheimer's disease: Prodromal metabolic phenotype and the search for genes. Hum Genomics 2010;4(3):170-93.

[14] Ridge PG, Curr Kauwe JSK. Mitochondria and Alzheimer's disease: the role of mitochondrial genetic variation. Genet Med Rep 2018;6(1):1/10.

[15] Berger AK, Fratiglioni L, Forsell Y, Winblad B, Backman L. The occurrence of depressive symptoms in the preclinical phase of AD: a population-based study. Neurol 1999;53(9):1998-2002.

[16] Devanand DP, Sano M, Tang MX, et al. Depressed mood and the incidence of Alzheimer's disease in the elderly living in the community. Arch Gen Psychiatry 1996;53(2):175-82.

[17] Kessing L, Andersen P. Does the risk of developing dementia increase with the number of episodes in patients with depressive disorder and in patients with bipolar disorder? J Neurol Neurosurg Psychiatry 2004;75(12):1662-6.

[18] Krishnan KRR, Tupler LA, Ritchie JC, McDonald WM, Knight DL, Nemeroff CB, et al.
Apolipoprotein E- e4 frequency in geriatric depression. Biol Psychiatry 1996;40:69-71.

[19] Ford DE, Erlinger TP. Depression and C-reactive protein in US adults: data from the Third National Health and Nutrition Examination Survey. Arch Intern Med 2004;164(9):1010-4.

[20] Pietrzak RH, Lim YY, Neumeister A, et al. Amyloid- $\beta$, anxiety, and cognitive decline in preclinical Alzheimer disease: a multicenter, prospective cohort study. JAMA Psychiatry 2015;72(3):284-91.

[21] Crocco E, Curiel RE, Acevedo A, Czaja SJ, Loewenstein DA. An evaluation of deficits in semantic cueing and proactive and retroactive interference as early features of Alzheimer's disease. Amer J Geriatric Psychiatry 2014;22(9):889-97.

[22] Abulafia C, Duarte-Abritta B, Villarreal MF, et al. Relationship between cognitive and sleep-wake variables in asymptomatic offspring of patients with Late-onset Alzheimer's disease. Front Aging Neurosci 2017;9(93):1-8.

[23] Folstein MF, Folstein SE, McHugh PR. Mini-mental state-A practical method for grading the cognitive state of patients for the clinician. J Psychiatry Research 1975;12:189-98.

[24] Solomon PR, Hirscho A, Kelly B, Relin M, Brush M, Deveaux RD. A 7 minute neuron cognitive screening battery highly sensitive to Alzheimer's disease. Arch Neurol 1998;55:349-55.

[25] Ravaglia G, Maioli F, Martelli M, Brunetti N, Bastagli L, Mariani E. Screening for mild cognitive impairment in elderly ambulatory patients with cognitive complaints. Aging Clin Exp Res 2005;17:374-9.

[26] Rey A. L'Examen Clinique en Psychologie. Paris: Presses Universitaires de France; 1964.

[27] Schmidt M. Rey auditory and verbal learning test: a handbook. Los Angeles, CA: Western Psychological Services; 1996.

[28] Spreen O, Benton AL. Neurosensory centre comprehensive examination for aphasia: manual of directions. Victoria, BC: University of Victoria, Neuropsychology Laboratory; 1969.

[29] Wechsler DA. Wechsler Adult Intelligence Scale-III. New York, NY: Psychological Corporation; 1997.

[30] Kaplan EF, Goodglass H, Weintraub S. Test de vocabulario de Boston. Madrid: Editorial Medica Panamericana; 1986.

[31] Harris P, Suarez MF, Surace EI, et al. Cognitive reserve and AßI-42 in mild cognitive impairment (Argentina-Alzheimer's Disease Neuroimaging Initiative). Neuropsychiatr Dis Treat 2015;11:2599-604.

[32] Artiola L, Hermosillo D, Heaton R, Pardee RE. Manual de normas y procedimientos para la batería neuropsicológica en español [Manual of norms and procedures for the Spanish neuropsychological battery] Tucson. AZ: M Press; 1999.

[33] Curiel RE, Crocco E, Acevedo A, Duara R, Agron J, Loewenstein DA. A new scale for the evaluation of proactive and retroactive interference in mild cognitive impairment and early Alzheimer's disease. J Aging Sci 2013;1:102.

[34] Rami L, Valls-Pedret C, Bartrés-Faz D, Caprile C, Solé-Padullés C, Castellvi M, et al. Cognitive reserve questionnaire-Scores obtained in a healthy elderly population and in one with Alzheimer's disease. Rev Neurologique 2011;52:195-201.

[35] Stern Y. Cognitive reserve in ageing and Alzheimer's disease. Lancet Neurol 2012;11(11):1006-12.

[36] Seed-based d Mapping Project web. FDR online calculator, http://www.sdmproject. com/utilities/?show =FDR [accessed July 2017].

[37] Murray J, et al. FDG and amyloid PET in cognitively normal individuals at risk for Late-onset Alzheimer's disease. Adv Mol Imaging 2014;4(2):15-26.

[38] Mosconi L, Brys M, Switalski R, Mistur R, et al. Maternal family history of Alzheimer's disease predisposes to reduced brain glucose metabolism. Proc Nat Acad Sci USA 2007;104:19067-72.

[39] Honea RA, Swerdlow RH, Vidoni ED, Burns JM. Progressive regional atrophy in normal adults with a maternal history of Alzheimer's disease. Neurol 2011;76(9):822-9.

[40] Heggeli K, Crook J, Thomas C, Graff-Radford N. Maternal transmission of Alzheimer disease. Alzheimer Dis Assoc Discord 2012;26(4):364-6.

[41] Abulafia C, Loewenstein DA, Curiel R, Duarte-Abritta B, Sánchez SM, Vigo DE, Castro MN, Drucaroff L, Vazquez S, Sevlever G, Nemeroff CB, Guinjoan SM, Villarreal MF. Brain Structural and Amyloid Correlates of Recovery from Semantic Interference in Cognitively Normal Individuals with or without Family History of Late-Onset Alzheimer's Disease. J Neuropsychiatry. Clin Neurosci In press.

[42] Sánchez SM, Abulafia C, Duarte-Abritta B, de Guevara MSL, Castro MN, Drucaroff L, et al. Failure to recover from proactive semantic interference and abnormal limbic connectivity in asymptomatic, middle-aged offspring of patients with Late-onset Alzheimer's disease. J Alzheimers Dis 2017;60(3):1183-93.

[43] Araujo NB, Moraes HS, Silveira H, et al. Impaired cognition in depression and Alzheimer (AD): a gradient from depression to depression in AD. Arq Neuropsiquiatry 2014;72(9):671-9. 